EPJ Web of Conferences 13, 05001 (2011)

DOI: $10.1051 /$ epjconf/20111305001

C Owned by the authors, published by EDP Sciences, 2011

\title{
Nonextensive statistical mechanics: Applications to high energy physics
}

\author{
Constantino Tsallis ${ }^{1,2}$ a
}

1 Centro Brasileiro de Pesquisas Fisicas and National Institute of Science and Technology for Complex Systems Rua Xavier Sigaud 150, 22290-180 Rio de Janeiro-RJ, Brazil

2 Santa Fe Institute, 1399 Hyde Park Road, Santa Fe, NM 87501, USA

\begin{abstract}
Nonextensive statistical mechanics was proposed in 1988 on the basis of the nonadditive entropy $S_{q}=k\left[1-\sum_{i} p_{i}^{q}\right] /(q-1)(q \in \mathcal{R})$ which generalizes that of Boltzmann-Gibbs $S_{B G}=S_{1}=-k \sum_{i} p_{i} \ln p_{i}$. This theory extends the applicability of standard statistical mechanics in order to also cover a wide class of anomalous systems which violate usual requirements such as ergodicity. Along the last two decades, a variety of applications have emerged in natural, artificial and social systems, including high energy phenomena. A brief review of the latter will be presented here, emphasizing some open issues.
\end{abstract}

\section{Introduction}

Standard statistical mechanics is based on the BoltzmannGibbs (BG) entropy $S_{B G}=-k \sum_{i=1}^{W} p_{i} \ln p_{i}\left(\sum_{i=1}^{W} p_{i}=\right.$ 1 ), where $W$ is the number of microscopic configurations of the system. This extremely powerful theory - one of the pillars of contemporary physics — has exhibited very many successes along 140 years, in particular through its celebrated distribution for thermal equilibrium $p_{i} \propto e^{-\beta E_{i}}$, $E_{i}$ being the energy of the corresponding microstate. However, as any other human intellectual construct, it has a restricted domain of validity. For nonlinear dynamical manybody systems the usual requirement is ergodicity, which is guaranted by strong chaos (i.e., by a positive maximal Lyapunov exponent for classical systems). For nonergodic systems (typically for systems whose maximal Lyapunov exponent vanishes), which is quite frequently the case of the so-called complex systems, there is no general reason for legitimately using the BG theory. For (some of) such anomalous systems, a generalization of the BG theory has been proposed in 1988 [1]. It is frequently referred to as nonextensive statistical mechanics [2-4] because the total energy of such systems typically is nonextensive, i.e., not proportional to the total number of elements of the system. This generalized theory is based on the entropy

$$
S_{q}=k \frac{1-\sum_{i} p_{i}^{q}}{q-1} \quad\left(q \in \mathcal{R} ; S_{1}=S_{B G}\right)
$$

It can be straightforwardly verified that, if $A$ and $B$ are two probabilistically independent systems (i.e., if $p_{i j}^{A+B}=$ $\left.p_{i}^{A} p_{j}^{B}\right)$, then

$$
\frac{S_{q}(A+B)}{k}=\frac{S_{q}(A)}{k}+\frac{S_{q}(B)}{k}+(1-q) \frac{S_{q}(A)}{k} \frac{S_{q}(B)}{k},
$$

\footnotetext{
a e-mail: tsallis@cbpf.br
}

which exhibits that, in contrast with $S_{B G}$ which is additive, the entropy $S_{q}$ is nonadditive for $q \neq 1$. This nonadditivity will in fact enable it to be extensive (i.e., proportional to the number of elements of the system) for various classes of systems (see for instance [5,6]).

\section{Connection to Thermodynamics}

To generalize BG statistical mechanics for the canonical ensemble (from [7]), we optimize $S_{q}$ with the constraints

$$
\sum_{i=1}^{W} p_{i}=1
$$

and

$$
\sum_{i=1}^{W} P_{i} E_{i}=U_{q}
$$

where

$$
P_{i} \equiv \frac{p_{i}^{q}}{\sum_{j=1}^{W} p_{i}^{q}}\left(\sum_{i=1}^{W} P_{i}=1\right)
$$

is the so-called escort distribution [8]. It follows that $p_{i}=$ $\frac{P_{i}^{1 / q}}{\sum_{j=1}^{W} P_{j}^{1 / q}}$. There are various converging reasons for being appropriate to impose the energy constraint with the $\left\{P_{i}\right\}$ instead of with the original $\left\{p_{i}\right\}$. The full discussion of this delicate point is beyond the present scope. However, some of these intertwined reasons are explored in [2]. By imposing Eq. (4), we follow [7], which in turn reformulates the results presented in $[1,9]$. The passage from one to the other of the various existing formulations of the above optimization problem are discussed in detail in $[7,10]$.

The entropy optimization yields, for the stationary state,

$$
p_{i}=\frac{e_{q}^{-\beta_{q}\left(E_{i}-U_{q}\right)}}{\bar{Z}_{q}},
$$

This is an Open Access article distributed under the terms of the Creative Commons Attribution-Noncommercial License 3.0, which permits unrestricted use, distribution, and reproduction in any noncommercial medium, provided the original work is properly cited. 
with

$$
\beta_{q} \equiv \frac{\beta}{\sum_{j=1}^{W} p_{j}^{q}},
$$

and

$$
\bar{Z}_{q} \equiv \sum_{i}^{W} e_{q}^{-\beta_{q}\left(E_{i}-U_{q}\right)},
$$

$\beta$ being the Lagrange parameter associated with the constraint (4). Eq. (6) makes explicit that the probability distribution is, for fixed $\beta_{q}$, invariant with regard to the arbitrary choice of the zero of energies. The stationary state (or (meta)equilibrium) distribution (6) can be rewritten as follows:

$$
p_{i}=\frac{e_{q}^{-\beta_{q}^{\prime} E_{i}}}{Z_{q}^{\prime}},
$$

with

$$
Z_{q}^{\prime} \equiv \sum_{j=1}^{W} e_{q}^{-\beta_{q}^{\prime} E_{j}}
$$

and

$$
\beta_{q}^{\prime} \equiv \frac{\beta_{q}}{1+(1-q) \beta_{q} U_{q}} .
$$

The form (9) is particularly convenient for many applications where comparison with experimental or computational data is involved. Also, it makes clear that $p_{i}$ asymptotically decays like $1 / E_{i}^{1 /(q-1)}$ for $q>1$, and has a cutoff for $q<1$, instead of the exponential decay with $E_{i}$ for $q=1$.

The connection to thermodynamics is established in what follows. It can be proved that

$$
\frac{1}{T}=\frac{\partial S_{q}}{\partial U_{q}},
$$

with $T \equiv 1 /(k \beta)$. Also we prove, for the free energy,

$$
F_{q} \equiv U_{q}-T S_{q}=-\frac{1}{\beta} \ln _{q} Z_{q},
$$

where

$$
\ln _{q} Z_{q}=\ln _{q} \bar{Z}_{q}-\beta U_{q} .
$$

This relation takes into account the trivial fact that, in contrast with what is usually done in BG statistics, the energies $\left\{E_{i}\right\}$ are here referred to $U_{q}$ in (6). It can also be proved

$$
U_{q}=-\frac{\partial}{\partial \beta} \ln _{q} Z_{q}
$$

as well as relations such as

$$
C_{q} \equiv T \frac{\partial S_{q}}{\partial T}=\frac{\partial U_{q}}{\partial T}=-T \frac{\partial^{2} F_{q}}{\partial T^{2}} .
$$

In fact, the entire Legendre transformation structure of thermodynamics is $q$-invariant, which is both remarkable and welcome.

\section{Applications}

\subsection{In diverse systems}

The nonadditive entropy $S_{q}$ and its associated nonetensive statistical mechanics have been applied to a wide variety of natural, artificial and social systems. Among others we may mention (i) The velocity distribution of (cells of) $\mathrm{Hy}$ dra viridissima follows a $q=3 / 2$ probability distribution function (PDF) [11]; (ii) The velocity distribution of (cells of) Dictyostelium discoideum follows a $q=5 / 3 \mathrm{PDF}$ in the vegetative state and a $q=2 \mathrm{PDF}$ in the starved state [12]; (iii) The velocity distribution in defect turbulence [13]; (iv) The velocity distribution of cold atoms in a dissipative optical lattice [14]; (v) The velocity distribution during silo drainage $[15,16]$; (vi) The velocity distribution in a drivendissipative 2D dusty plasma, with $q=1.08 \pm 0.01$ and $q=1.05 \pm 0.01$ at temperatures of $30000 \mathrm{~K}$ and $61000 \mathrm{~K}$ respectively [17]; (vii) The spatial (Monte Carlo) distributions of a trapped ${ }^{136} \mathrm{Ba}^{+}$ion cooled by various classical buffer gases at $300 K$ [18]; (viii) The distributions of price returns and stock volumes at the stock exchange, as well as the volatility smile [19-22]; (ix) Biological evolution [23]; (x) The distributions of returns in the Ehrenfest's dog-flea model [24,25]; (xi) The distributions of returns in the coherent noise model [26]; (xii) The distributions of returns of the avalanche sizes in the self-organized critical OlamiFeder-Christensen model, as well as in real earthquakes [27]; (xiii) The distributions of angles in the $H M F$ model [28]; (xiv) Turbulence in electron plasma [29]; (xv) The relaxation in various paradigmatic spin-glass substances through neutron spin echo experiments [30]; (xvi) Various properties directly related with the time dependence of the width of the ozone layer around the Earth [31]; (xvii) Various properties for conservative and dissipative nonlinear dynamical systems [32-41]; (xviii) The degree distribution of (asymptotically) scale-free networks [42,43]; (xix) Tissue radiation response [44]; $(\mathrm{xx})$ Overdamped motion of interacting particles [45]; (xxi) Rotational population in molecular spectra in plasmas [46]. The systematic study of metastable or long-living states in long-range versions of magnetic models such as the Ising [47] and Heisenberg [48] ones, or in hydrogen-like atoms [49-51] might provide further illustrations.

\subsection{In high energy physics}

Connections of nonextensive statistics with a specific area of solar physics, astrophysics, high energy physics, and related areas, were pioneered by Quarati and collaborators (see [52], among others), who advanced the possibility of this theory being useful in the discussion of the flux of solar neutrinos. A few years later, it was realized that the transverse momenta distribution of the hadronic jets resulting from electron-positron annihilation are well described by distributions associated with $q$-exponentials $[53,54]$ : see Figs. 1 and 2. The energy distribution of cosmic rays has been satisfactorily fitted in $[55,56]$ with distributions related to $q$-exponentials: see Fig. 3. The distributions of returns of magnetic field fluctuations in the solar 
wind plasma as observed in data from Voyager 1 [57] and from Voyager 2 [58] has provided the values associated with the so called $q$-triplet: see Figs. 4 e 5. Similar results have been obtained in the study of interstellar turbulence [59] (see Figs. 6 and 7), in X-ray-emitting binary systems [60] (see Fig. 8), and in the distribution of stellar rotational velocities in the Pleiades [61].

It is important to address here the fact that the distribution of transverse momenta in high-energy collisions of proton-proton, and heavy nuclei (e.g., $\mathrm{Pb}-\mathrm{Pb}$ and $\mathrm{Au}-\mathrm{Au}$ ) have received and are receiving great attention [62-68]: see illustrative examples in Figs. 9-15. Several such data have been summarized in [69]: see Fig. 16. We realize that for such collisions the typical values of $q$ are usually close to 1.10 , apparently never above say $1.20-1.25$. It remains as a challenging problem to precisely understand why (Is it a hadronization of quark matter in a sort of metastable state before attaining ergodicity?). In any case, it was shown in [71] that QCD calculations and $q$-statistical calculations can be consistent for $q \simeq 1.1$ : see Fig. 17 .

\section{Acknowledgments}

I gratefully acknowledge wonderful hospitality received in Budapest at the occasion of the HCBM meeting, very especially from Prof. T.S. Biro. Naturally, the authors of the various figures that $I$ have reproduced in the present brief review are acknowledged as well.

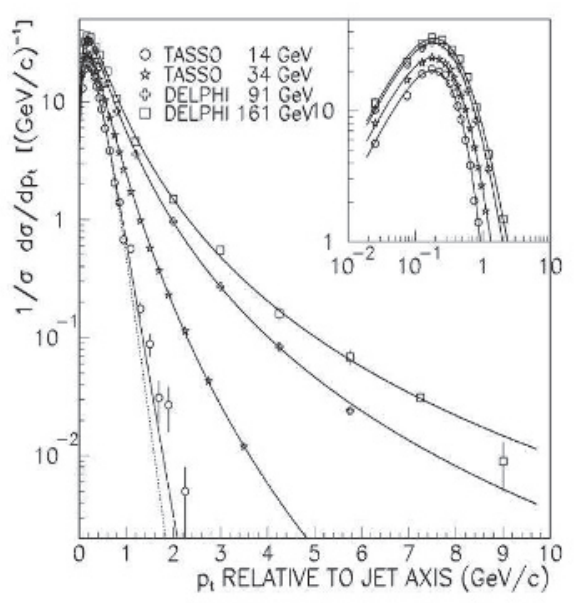

Fig. 1. Distributions of transverse momenta for four typical values of the collision energy. See details in [53].

\section{References}

1. C. Tsallis, Possible generalization of BoltzmannGibbs statistics, J. Stat. Phys. 52 (1988) 479-487.

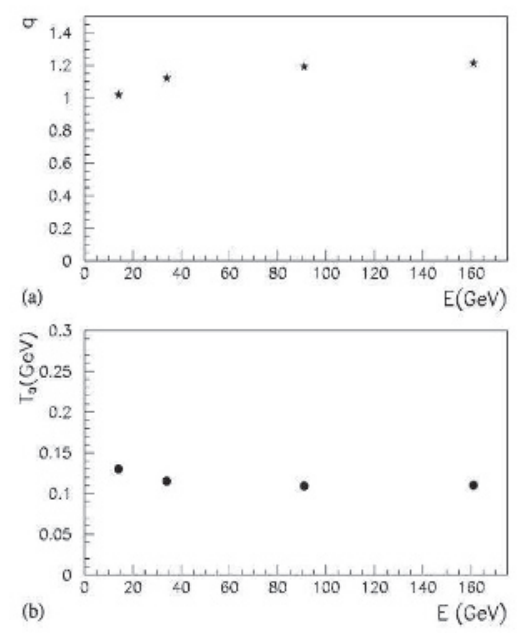

Fig. 2. Dependence of the index $q$ (a) and the temperature $T_{0}$ (b) on the collision energies of Fig. 3. The particular case $q=1$ corresponds to the Hagedorn 1965 theory. It is advanced in [54] the possibility that $q$ approaches the value $11 / 9$ in the $E \rightarrow \infty$ limit. See details in [53].

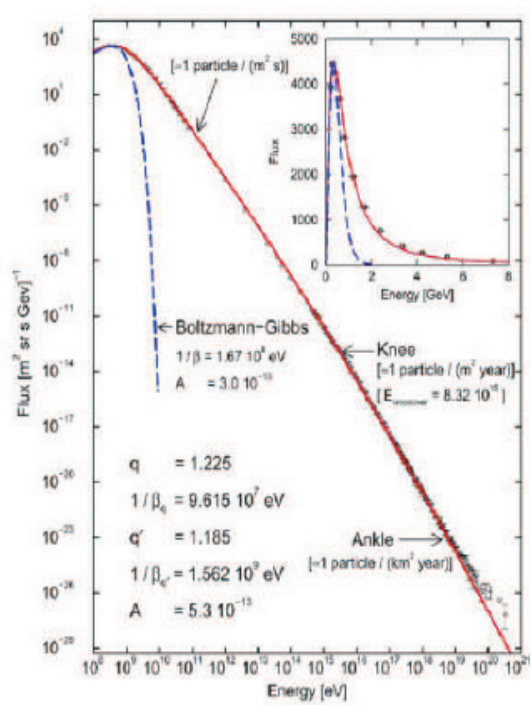

Fig. 3. Flux of cosmic rays. Curiously enough, the upper value of the index $q$ is very close to $11 / 9$. See details in $[55,56]$.

2. C. Tsallis, Introduction to Nonextensive Statistical Mechanics - Approaching a Complex World (Springer, New York, 2009).

3. C. Tsallis, Entropy, in Encyclopedia of Complexity and Systems Science, ed. R.A. Meyers (Springer, Berlin, 2009), 11 volumes [ISBN: 978-0-387-758886].

4. A regularly updated bibliography can be seen at http://tsallis.cat.cbpf.br/biblio.htm

5. F. Caruso and C. Tsallis, Nonadditive entropy reconciles the area law in quantum systems with classical 


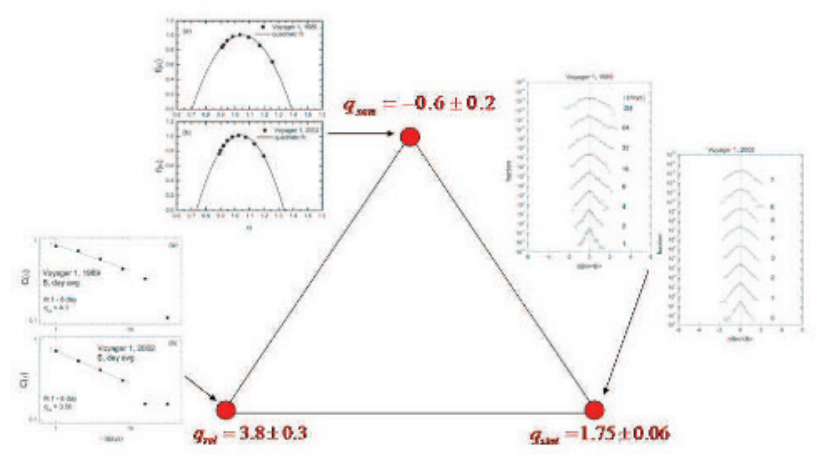

Fig. 4. The $q$-triplet as obtained from data of the Voyager 1. See details in [57].

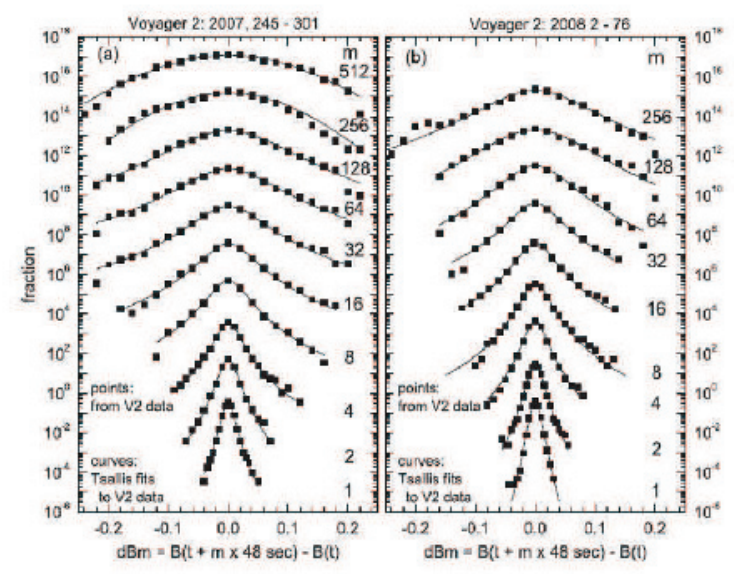

Fig. 5. Distributions from which $q_{\text {stat }}$ is extracted, from data of the Voyager 2. See details in [58].

thermodynamics, Phys. Rev. E 78 (2008) 021101.

6. A. Saguia and M.S. Sarandy, Nonadditive entropy for random quantum spin-S chains, Phys. Lett. A 374, 3384-3388 (2010).

7. C. Tsallis, R.S. Mendes and A.R. Plastino, The role of constraints within generalized nonextensive statistics, Physica A 261 (1998) 534-554.

8. C. Beck and F. Schlogl, Thermodynamics of Chaotic Systems (Cambridge University Press, Cambridge, 1993).

9. E.M.F. Curado and C. Tsallis, Generalized statistical mechanics: connection with thermodynamics, J. Phys. A 24, L69 (1991); Corrigenda: 24, 3187 (1991) and 25, 1019 (1992).

10. G.L. Ferri, S. Martinez and A. Plastino, Equivalence of the four versions of Tsallis' statistics, JSTAT- Journal of Statistical Mechanics: Theory and Experiment 1742-5468/05/P04009 (2005).

11. A. Upadhyaya, J.-P. Rieu, J.A. Glazier and Y. Sawada, Anomalous diffusion and non-Gaussian velocity dis-

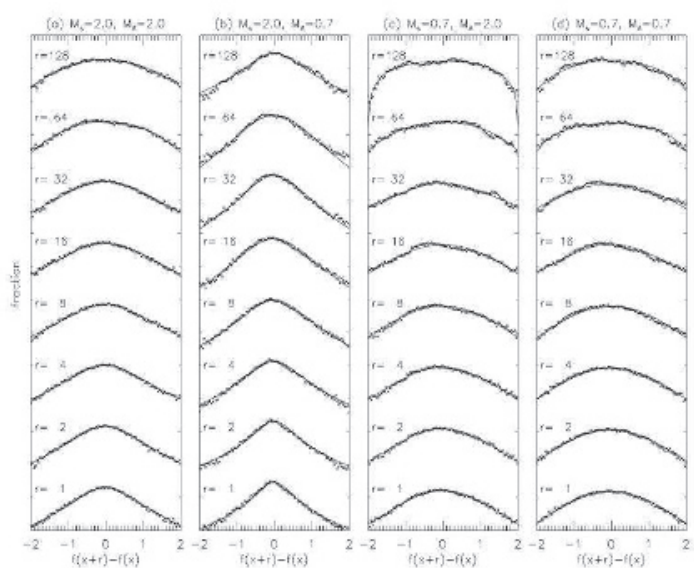

Fig. 6. Distributions of column density fluctuations for different spatial separations. See details in [59].

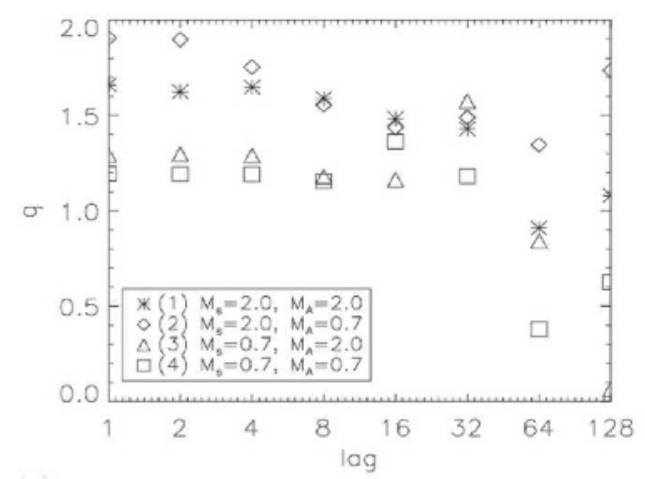

Fig. 7. Values of the index $q_{\text {stat }}$ corresponding to Fig. 6. See details in [59].

tribution of Hydra cells in cellular aggregates, Physica A 293 (2001) 549.

12. A.M. Reynolds, Can spontaneous cell movements be modelled as Lévy walks?, Physica A 389, 273 (2010).

13. K.E. Daniels, C. Beck and E. Bodenschatz, Defect turbulence and generalized statistical mechanics, Physica D 193, 208 (2004).

14. P. Douglas, S. Bergamini and F. Renzoni [2006], Tunable Tsallis distributions in dissipative optical lattices, Phys. Rev. Lett. 96, 110601; G.B. Bagci and U. Tirnakli [2009], Self-organization in dissipative optical lattices, Chaos 19, 033113.

15. R. Arevalo, A. Garcimartin and D. Maza, Anomalous diffusion in silo drainage, Eur. Phys. J. E 23, 191-198 (2007).

16. R. Arevalo, A. Garcimartin and D. Maza, A nonstandard statistical approach to the silo discharge, in Complex Systems - New Trends and Expectations, eds. H.S. Wio, M.A. Rodriguez and L. Pesquera, Eur. Phys. 

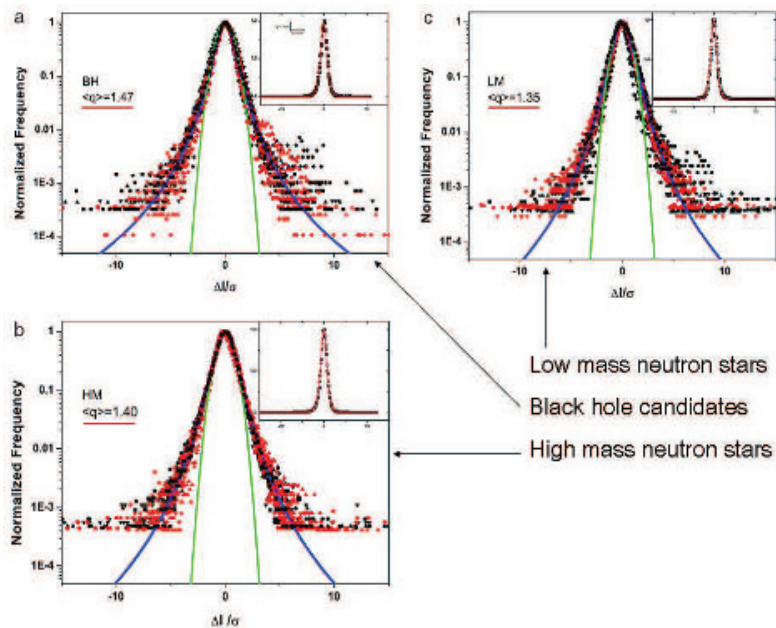

Low mass neutron stars

Black hole candidates

High mass neutron stars

Fig. 8. Distributions of intensities of X-ray emission. See details in $[60]$.
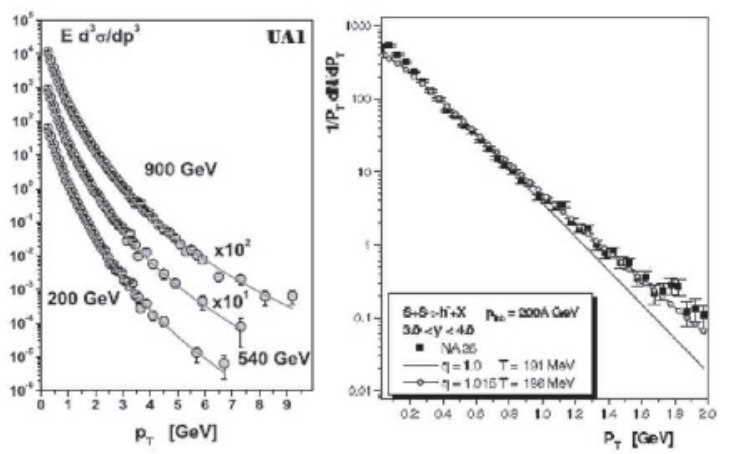

Fig. 9. Transverse momenta distributions for different energies. See details in [62].

J.-Special Topics 143 (2007).

17. B. Liu and J. Goree [2008], Superdiffusion and nonGaussian statistics in a driven-dissipative $2 D$ dusty plasma, Phys. Rev. Lett. 100, 055003.

18. R.G. DeVoe, Power-law distributions for a trapped ion interacting with a classical buffer gas, Phys. Rev. Lett. 102, 063001 (2009).

19. L. Borland, Closed form option pricing formulas based on a non-Gaussian stock price model with statistical feedback, Phys. Rev. Lett. 89, 098701 (2002).

20. L. Borland, A theory of non-gaussian option pricing, Quantitative Finance 2, 415 (2002).

21. R. Osorio, L. Borland and C. Tsallis, Distributions of high-frequency stock-market observables, in Nonextensive Entropy - Interdisciplinary Applications, eds. M. Gell-Mann and C. Tsallis (Oxford University Press, New York, 2004).

22. S.M.D. Queiros, On non-Gaussianity and dependence in financial in time series: A nonextensive approach, Quant. Finance 5, 475 (2005).

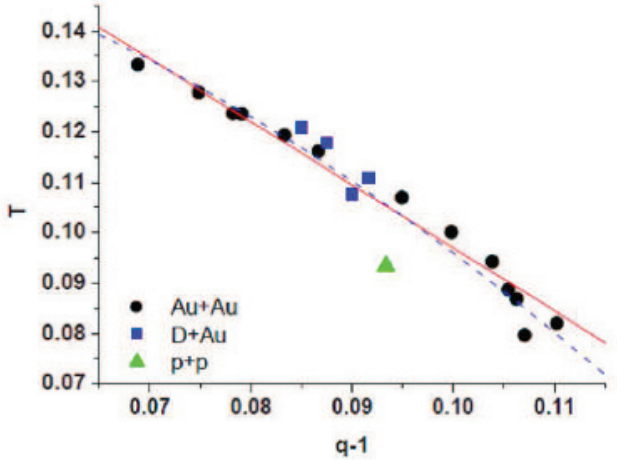

Fig. 10. Dependence of the temperature $T$ on the index $q$ for production of negative pions in different reactions. See details in [62].

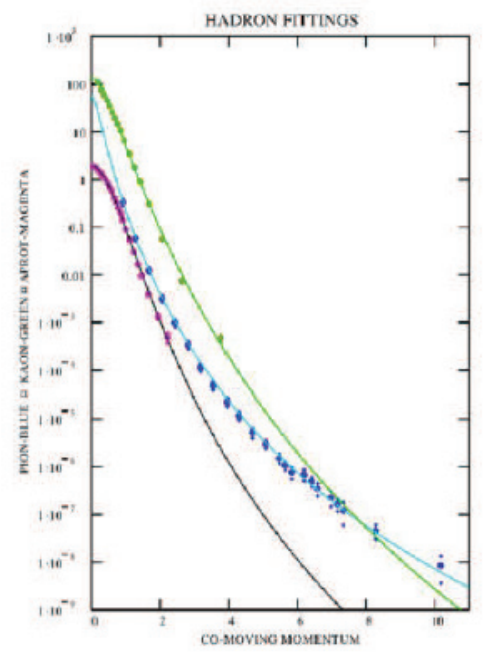

Fig. 11. Transverse momenta spectra for pions, kaons and antiprotons in relativistic heavy ions experiments. See details in [63].

23. F.A. Tamarit, S.A. Cannas and C. Tsallis, Sensitivity to initial conditions in the Bak-Sneppen model of biological evolution, Eur. Phys. J. B 1, 545 (1998).

24. B. Bakar and U. Tirnakli, Analysis of self-organized criticality in Ehrenfest's dog-flea model, Phys. Rev. E 79, 040103(R) (2009).

25. B. Bakar and U. Tirnakli, Return distributions in dogflea model revisited, Physica A 389, 3382 (2010).

26. A. Celikoglu, U. Tirnakli and S.M.D. Queiros, Analysis of return distributions in the coherent noise model, Phys. Rev. E 82, 021124 (2010).

27. F. Caruso, A. Pluchino, V. Latora, S. Vinciguerra and A. Rapisarda, Analysis of self-organized criticality in the Olami-Feder-Christensen model and in real earthquakes, Phys. Rev. E 75, 055101(R) (2007). 

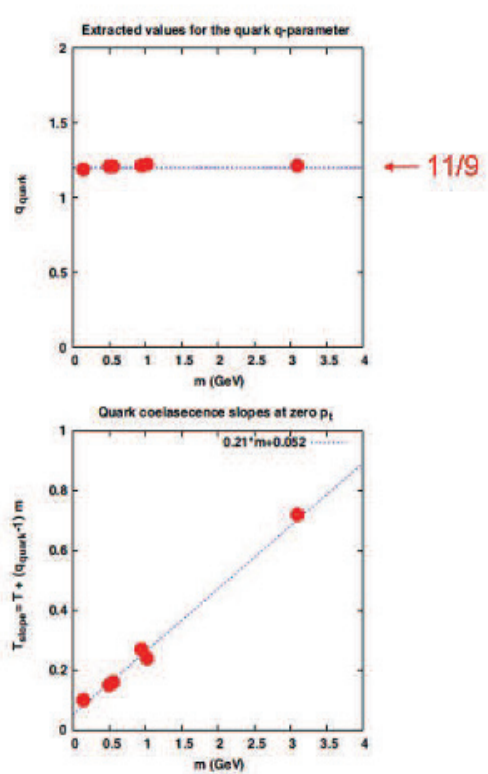

Fig. 12. The index $q$ (top) and the temperature (bottom) extracted from hadronic spectra assuming quark coalescence at a sudden hadron formation. See details in [63].

28. L.G. Moyano and C. Anteneodo, Diffusive anomalies in a long-range Hamiltonian system, Phys. Rev. E 74, 021118 (2006).

29. C. Anteneodo and C. Tsallis, Two-dimensional turbulence in pure-electron plasma: A nonextensive thermostatistical description, J. Molecular Liquids 71, 255 (1997).

30. R.M. Pickup, R. Cywinski, C. Pappas, B. Farago and P. Fouquet [2009], Generalized spin glass relaxation, Phys. Rev. Lett. 102, 097202.

31. G.L. Ferri, M.F. Reynoso Savio and A. Plastino, Tsallis? q-triplet and the ozone layer, Physica A 389, 1829 (2010).

32. M.L. Lyra and C. Tsallis, Nonextensivity and multifractality in low-dimensional dissipative systems, Phys. Rev. Lett. 80, 53 (1998).

33. U. Tirnakli, C. Tsallis and M.L. Lyra, Circular-like maps: Sensitivity to the initial conditions, multifractality and nonextensivity, Eur. Phys. J. B 11, 309 (1999).

34. E.P. Borges, C. Tsallis, G.F.J. Ananos and P.M.C. Oliveira, Nonequilibrium probabilistic dynamics at the logistic map edge of chaos, Phys. Rev. Lett. 89, 254103 (2002).

35. G.F.J. Ananos and C. Tsallis, Ensemble averages and nonextensivity at the edge of chaos of one-dimensional maps, Phys. Rev. Lett. 93, 020601 (2004).

36. F. Baldovin and A. Robledo, Nonextensive Pesin identity. Exact renormalizat-

ion group analytical results for the dynamics at the edge of chaos of the logistic map, Phys. Rev. E 69, 045202(R) (2004).
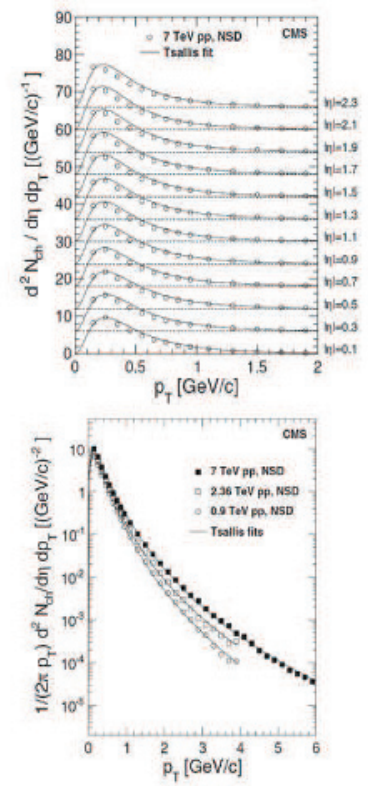

Fig. 13. Transverse momenta distributions of charged hadrons in pp collisions, as measured by the CMS Collaboration at LHC, corresponding to $0.9,2.36$ and $7 \mathrm{TeV}$. At these energies it has been obtained $(q, T)=(1.13,0.13 \mathrm{GeV}),(1.15,0.14 \mathrm{GeV}),(1.15, o .145 \mathrm{GeV})$ respectively. See details in $[64,65]$.

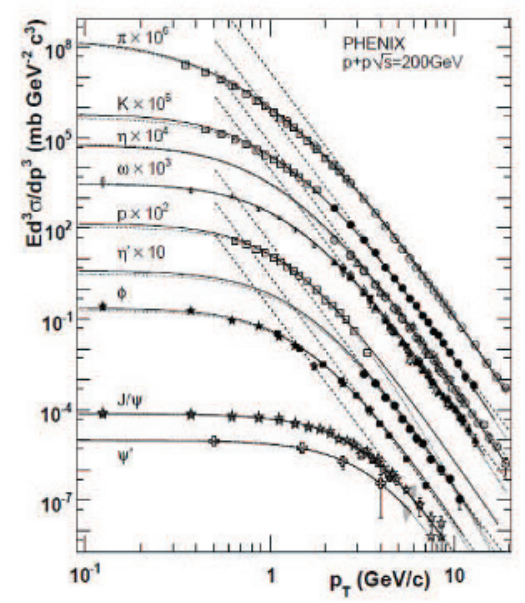

Fig. 14. Transverse momenta distributions of various hadrons in pp collisions, as measured by the PHENIX Collaboration, corresponding to $200 \mathrm{GeV}$. At this energy it has been obtained $q \simeq 1.10$. See details in [67].

37. E. Mayoral and A. Robledo, Tsallis' $q$ index and Mori's q phase transitions at edge of chaos, Phys. Rev. E 72, 026209 (2005).

38. A. Pluchino, A. Rapisarda and C. Tsallis, Nonergodicity and central limit behavior in long-range Hamiltonians, Europhys. Lett. 80, 26002 (2007). 

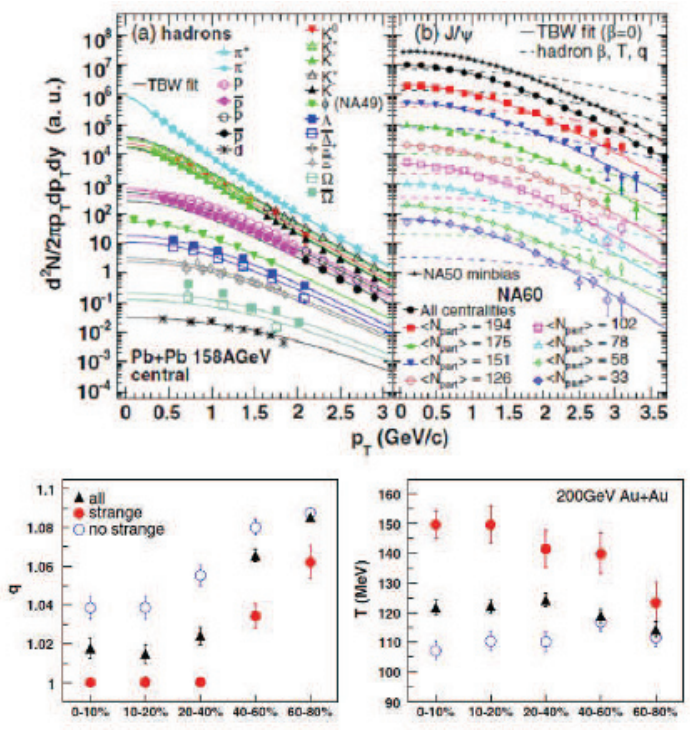

Fig. 15. Transverse momenta distributions of charged hadrons in $\mathrm{pp}$ and heavy ion collisions, as measured in Brookhaven. See details in [68].

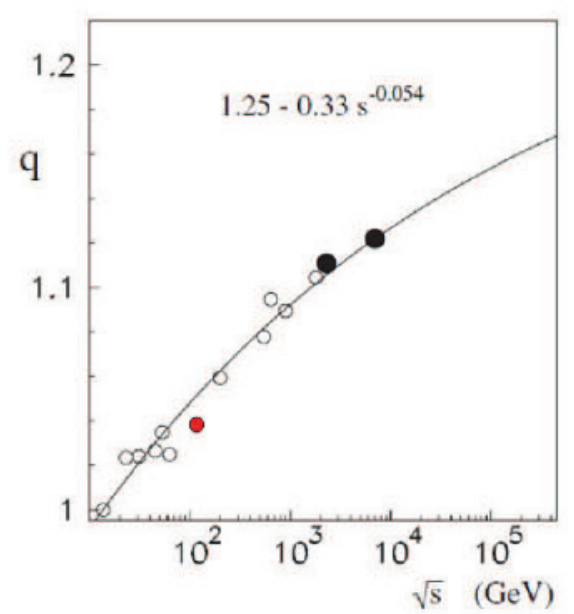

Fig. 16. Index $q$ obtained at various energies. See details in [69]. The black dots indicate recent CMS results. The red dot indicates the value obtained in [70].

39. A. Pluchino, A. Rapisarda and C. Tsallis, A closer look at the indications of q-generalized Central Limit Theorem behavior in quasi-stationary states of the HMF model, Physica A 387, 3121 (2008).

40. G. Miritello, A. Pluchino and A. Rapisarda, Central limit behavior in the Kuramoto model at the 'edge of chaos', Physica A 388, 4818 (2009).

41. M. Leo, R.A. Leo and P. Tempesta, Thermostatistics in the neighborhood of the $\pi$-mode solution for the Fermi-Pasta-Ulam $\beta$ system: From weak to strong chaos, J. Stat. Mech. P04021 (2010).

42. D.R. White, N. Kejzar, C. Tsallis,D. Farmer and S. White, A generative model for feedback networks,

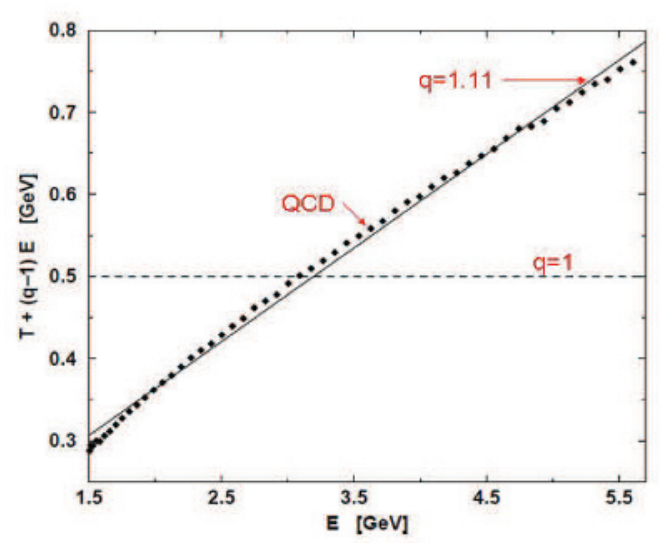

Fig. 17. Comparison of QCD diffusion calculation with its corresponding within $q$-statistics: they are consistent for $q=1.11$. See details in [71].

Phys. Rev. E 73, 016119 (2006).

43. S. Thurner, F. Kyriakopoulos and C. Tsallis [2007], Unified model for network dynamics exhibiting nonextensive statistics, Phys. Rev. E 76, 036111.

44. O. Sotolongo-Grau, D. Rodriguez-Perez, J.C. Antoranz and O. Sotolongo-Costa [2010], Tissue radiation response with maximum Tsallis entropy, Phys. Rev. Lett. 105, 158105 (4 pages).

45. J. S. Andrade Jr., G.F.T. da Silva, A.A. Moreira, F.D. Nobre and E.M.F. Curado [2010], Thermostatistics of overdamped motion of interacting particles, Phys. Rev. Lett. 105, 260601.

46. J.L. Reis Jr., J. Amorim and A. Dal Pino Jr., Occupancy of rotational population in molecular spectra based on nonextensive statistics, Phys. Rev. E 83, 017401 (2011) (4 pages).

47. F.D. Nobre and C. Tsallis, Infinite-range Ising ferromagnet: thermodynamic limit within generalized statistical mechanics, Physica A 213, 337 (1995); Erratum: 216, 369 (1995).

48. A.O. Caride, C. Tsallis and S. I. Zanette, Criticality of the anisotropic quantum Heisenberg model on a self-dual hierarchical lattice, Phys. Rev. Lett. 51, 145 (1983); 51, 616 (1983).

49. L.S. Lucena, L.R. da Silva and C. Tsallis, Departure from Boltzmann-Gibbs statistics makes the hydrogenatom specific heat a computable quantity, Phys. Rev. E 51, 6247 (1995).

50. N.M. Oliveira-Neto, E.M.F. Curado, F.D. Nobre and M.A. Rego-Monteiro, Approach to equilibrium of the hydrogen atom at low temperature, Physica A 374, 251-262 (2007).

51. N.M. Oliveira-Neto, E.M.F. Curado, F.D. Nobre and M.A. Rego-Monteiro, A simple model to describe the low-temperature behaviour of some atoms and molecules: An application to the hydrogen atom, J. 
Phys. B - Atomic, Molecular and Optical Physics 40, 1975-1989 (2007).

52. G. Kaniadakis, A. Lavagno and P. Quarati, Generalized statistics and solar neutrinos, Phys. Lett. B 369 , 308 (1996).

53. I. Bediaga, E.M.F. Curado and J. Miranda, A nonextensive thermodynamical equilibrium approach in $e^{+} e^{-} \rightarrow$ hadrons, Physica A 286, 156 (2000).

54. C. Beck, Non-extensive statistical mechanics and particle spectra in elementary interactions, Physica A 286, 164 (2000).

55. C. Tsallis, J.C. Anjos and E.P. Borges, Fluxes of cosmic rays: A delicately balanced stationary state, Phys. Lett. A 310, 372 (2003).

56. C. Beck, Generalized statistical mechanics of cosmic rays, Physica A 331, 173 (2003).

57. L.F. Burlaga and A.F.-Vinas, Triangle for the entropic index $q$ of non-extensive statistical mechanics observed by Voyager 1 in the distant heliosphere, Physica A 356, 375 (2005).

58. L.F. Burlaga and N.F. Ness, Compressible "turbulence" observed in the heliosheath by Voyager 2, Astrophys. J. 703, 311 (2009).

59. A. Esquivel and A. Lazarian, Tsallis statistics as a tool for studying interstellar turbulence, Astrophys. J. 710, 125-132 (2010).

60. M.A. Moret, V. de Senna, G.F. Zebende and P. Vaveliuk, X-ray binary systems and nonextensivity, Physica A 389, 854-858 (2010).

61. J.C. Carvalho, R. Silva, J.D. do Nascimento and J.R. de Medeiros, Power law statistics and stellar rotational velocities in the Pleiades, Europhys. Lett. 84, 59001 (2008).

62. G. Wilk and Z. Wlodarczyk, Power laws in elementary and heavy-ion collisions - A story of fluctuations and nonextensivity?, Eur. Phys. J. A 40, 299 (2009).

63. T.S. Biro, G. Purcsel and K. Urmossy, Non-extensive approach to quark matter, in Statistical Power-Law Tails in High Energy Phenomena, Eur. Phys. J. A 40, 325 (2009).

64. V. Khachatryan et al (CMS Collaboration), Transverse-momentum and pseudorapidity distributions of charged hadrons in pp collisions at $\sqrt{s}=0.9$ and $2.36 T e V$, J. High Energy Phys. 02, 041 (2010).

65. V. Khachatryan et al (CMS Collaboration), Transverse-momentum and pseudorapidity distributions of charged hadrons in pp collisions at $\sqrt{s}=7$ TeV, Phys. Rev. Lett. 105, 022002 (2010).

66. D. d'Enterria, R. Engel, T. Pierog, S. Ostapchenko and K. Werner, Constraints from the first LHC data on hadronic event generators for ultra-high energy cosmic-ray physics, 1101.5596 [astro-ph.HE] (2011).

67. Adare et al (PHENIX Collaboration), Measurement of neutral mesons in $p+p$ collisions at $\sqrt{s}=$ $200 \mathrm{GeV}$ and scaling properties of hadron production, 1005.3674 [hep-ex] (2010).

68. M. Shao, L. Yi, Z.B. Tang, H.F. Chen, C. Li and Z.B. $\mathrm{Xu}$, Examination of the species and beam energy dependence of particle spectra using Tsallis statistics, J.
Phys. G 37 (8), 085104 (2010).

69. T. Wibig, The non-extensivity parameter of a thermodynamical model of hadronic interactions at LHC energies, J. Phys. G: Nucl. Part. Phys. 37, 115009 (2010) (4 pages).

70. W.M. Alberico, A. Lavagno and P. Quarati, Nonextensive statistics, fluctuations and correlations in high energy nuclear collisions, Eur. Phys. J C 12, 499 (2000).

71. D.B. Walton and J. Rafelski, Equilibrium distribution of heavy quarks in Fokker-Planck dynamics, Phys. Rev. Lett. 84, 31 (2000). 